Proyecciones Journal of Mathematics

Vol. 33, No 2, pp. 175-187, June 2014.

Universidad Católica del Norte

Antofagasta - Chile

\title{
Upper Edge Detour Monophonic Number of a Graph
}

\author{
P. Titus \\ University College of Engineering Nagercoil, India \\ and \\ K. Ganesamoorthy \\ University V. O. C. College of Engineering Tuticorin, India \\ Received : November 2013. Accepted : March 2014
}

\begin{abstract}
For a connected graph $G$ of order at least two, a path $P$ is called a monophonic path if it is a chordless path. A longest $x-y$ monophonic path is called an $x-y$ detour monophonic path. A set $S$ of vertices of $G$ is an edge detour monophonic set of $G$ if every edge of $G$ lies on a detour monophonic path joining some pair of vertices in $S$. The edge detour monophonic number of $G$ is the minimum cardinality of its edge detour monophonic sets and is denoted by edm $(G)$. An edge detour monophonic set $S$ of $G$ is called a minimal edge detour monophonic set if no proper subset of $S$ is an edge detour monophonic set of $G$. The upper edge detour monophonic number of $G$, denoted by edm ${ }^{+}(G)$, is defined as the maximum cardinality of a minimal edge detour monophonic set of $G$. We determine bounds for it and characterize graphs which realize these bounds. For any three positive integers $b, c$ and $n$ with $2 \leq b \leq n \leq c$, there is a connected graph $G$ with $\operatorname{edm}(G)=b, e^{+} m^{+}(G)=c$ and a minimal edge detour monophonic set of cardinality $n$.
\end{abstract}

Key Words: edge detour monophonic set, edge detour monophonic number, minimal edge detour monophonic set, upper edge detour monophonic set, upper edge detour monophonic number.

AMS Subject Classification: 05C12. 


\section{Introduction}

By a graph $G=(V, E)$ we mean a finite undirected connected graph without loops or multiple edges. The order and size of $G$ are denoted by $p$ and $q$, respectively. For basic graph theoretic terminology we refer to Harary [1]. For vertices $x$ and $y$ in a connected graph $G$, the distance $d(x, y)$ is the length of a shortest $x-y$ path in $G$. An $x-y$ path of length $d(x, y)$ is called an $x-y$ geodesic. The neighborhood of a vertex $v$ is the set $N(v)$ consisting of all vertices $u$ which are adjacent with $v$. A vertex $v$ is an extreme vertex if the subgraph induced by its neighbors is complete. A vertex $v$ is a semi-extreme vertex of $G$ if the subgraph $G[S]$ induced by its neighborhood $S$ has a vertex with degree equal to $|S|-1$. In particular, every extreme vertex is a semi-extreme vertex and a semi-extreme vertex need not be an extreme vertex.

A chord of a path $P$ is an edge joining two non-adjacent vertices of $P$. A path $P$ is called monophonic if it is a chordless path. A longest $x-y$ monophonic path is called an $x-y$ detour monophonic path. A set $S$ of vertices of a graph $G$ is a detour monophonic set if each vertex $v$ of $G$ lies on an $x-y$ detour monophonic path for some $x, y \in S$. The cardinality of a detour monophonic set of $G$ with minimum cardinality is the detour monophonic number of $G$ and is denoted by $d m(G)$. The detour monophonic number of a graph was introduced in [4] and further studied in $[5]$.

An edge monophonic set of $G$ is a set $S$ of vertices such that every edge of $G$ lies on a monophonic path joining some pair of vertices in $S$. The edge monophonic number of $G$ is the minimum cardinality of its edge monophonic sets and is denoted by $m_{1}(G)$. An edge monophonic set of cardinality $m_{1}(G)$ is an $m_{1}$-set of $G$. An edge detour monophonic set of $G$ is a set $S$ of vertices such that every edge of $G$ lies on a detour monophonic path joining some pair of vertices in $S$. The edge detour monophonic number of $G$ is the minimum cardinality of its edge detour monophonic sets and is denoted by edm $(G)$. An edge detour monophonic set of cardinality $e d m(G)$ is an $e d m$-set of $G$. The edge detour monophonic number of a graph was introduced and studied in [3].

The following theorems will be used in the sequel.

Theorem 1.1. [2] Each semi-extreme vertex of a graph $G$ belongs to every edge monophonic set of $G$.

Theorem 1.2. [3] Each semi-extreme vertex of a graph $G$ belongs to every edge detour monophonic set of $G$. 
Theorem 1.3. [3] Let $G$ be a connected graph with cut-vertices and $S$ an edge detour monophonic set of $G$. If $v$ is a cut-vertex of $G$, then every component of $G-v$ contains an element of $S$.

Theorem 1.4. [3] For any connected graph $G$, no cut-vertex of $G$ belongs to any minimum edge detour monophonic set of $G$.

Theorem 1.5. [3] If $T$ is a tree with $k$ end-vertices, then $m_{1}(T)=e d m(T)=$ $k$.

Throughout this paper $G$ denotes a connected graph with at least two vertices.

\section{Upper edge detour monophonic number}

Definition 2.1. Let $G$ be a connected graph with at least two vertices. An edge detour monophonic set $S$ of $G$ is called a minimal edge detour monophonic set if no proper subset of $S$ is an edge detour monophonic set of $G$. The upper edge detour monophonic number of $G$, denoted by $e d m^{+}(G)$, is defined as the maximum cardinality of a minimal edge detour monophonic set of $G$.

Example 2.2. For the graph $G$ given in Figure 2.1, the minimal edge detour monophonic sets are $S_{1}=\{x, z\}$ and $S_{2}=\{y, u, v\}$. Hence the upper edge detour monophonic number of $G$ is 3 .

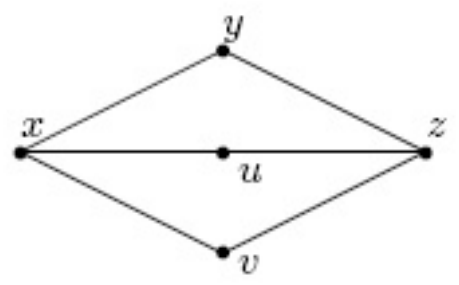

Figure 2.1: $G$ 
Note 2.3 Every minimum edge detour monophonic set is a minimal edge detour monophonic set, but the converse is not true. For the graph $G$ given in Figure 2.1, $S_{2}$ is a minimal edge detour monophonic set but it is not a minimum edge detour monophonic set of $G$.

Since every minimal edge detour monophonic set of $G$ is an edge detour monophonic set of $G$, we have the following theorems.

Theorem 2.3. Each semi-extreme vertex of a connected graph $G$ belongs to every minimal edge detour monophonic set of $G$.

Proof. This follows from Theorem 1.2.

Corollary 2.4. For the complete graph $K_{p}, e d m^{+}\left(K_{p}\right)=p$.

Proof. Since every vertex of $K_{p}$ is a semi-extreme vertex, the result follows from Theorem 2.3.

Theorem 2.5. Let $G$ be a connected graph with cut-vertices and let $S$ be a minimal edge detour monophonic set of $G$. If $v$ is a cut-vertex of $G$, then every component of $G-v$ contains an element of $S$.

Proof. This follows from Theorem 1.3.

Corollary 2.6. Let $G$ be a connected graph with cut-vertices and let $S$ be a minimal edge detour monophonic set of $G$. Then every branch of $G$ contains an element of $S$.

Proof. This follows from Theorem 2.5.

Theorem 2.7. No cut-vertex of a connected graph $G$ belongs to any minimal edge detour monophonic set of $G$.

Proof. This follows from Theorem 1.4.

Corollary 2.8. For any tree $T$ with $k$ end-vertices, $e d m(T)=e d m^{+}(T)=$ $k$. 
Proof. Since every vertex of $T$ is either a semi-extreme vertex or a cut-vertex, the result follows from Theorems 2.3 and 2.7 .

We denote the vertex connectivity of a connected graph $G$ by $\kappa(G)$ or $\kappa$.

Theorem 2.9. If $G$ is a non-complete connected graph such that it has a minimum cut set consisting of $\kappa$ vertices, then $e d m^{+}(G) \leq p-\kappa$.

Proof. Since $G$ is a non-complete connected graph, it is clear that $1 \leq \kappa \leq p-2$. Let $U=\left\{u_{1}, u_{2}, u_{3}, \ldots, u_{\kappa}\right\}$ be a minimum cut set of $G$. Let $G_{1}, G_{2}, \ldots, G_{r}(r \geq 2)$ be the components of $G-U$ and let $S=V(G)-U$. Then every vertex $u_{i}(1 \leq i \leq \kappa)$ is adjacent to at least one vertex of $G_{j}$ for each $j(1 \leq j \leq r)$. It is clear that $S$ is an edge detour monophonic set of $G$ and so $e d m^{+}(G) \leq|S|=p-\kappa$.

Remark 2.10. The bound in Theorem 2.9 is sharp for the graph $G$ given in Figure 2.1.

Theorem 2.11. For any connected graph $G, 2 \leq e d m(G) \leq e d m^{+}(G) \leq$ $p$.

Proof. It is clear from the definition of minimum edge detour monophonic set that $\operatorname{edm}(G) \geq 2$. Since every minimal edge detour monophonic set is an edge detour monophonic set of $G, e d m(G) \leq e d m^{+}(G)$. Also, since $V(G)$ induces an edge detour monophonic set of $G$, it is clear that $e d m^{+}(G) \leq p$. Thus $2 \leq e d m(G) \leq e d m^{+}(G) \leq p$.

Remark 2.12. The bounds in Theorem 2.11 are all sharp for $K_{2}$. Furthermore, for any tree $T$ with $k$ end-vertices $e d m(T)=e d m^{+}(T)=k$ (notice that a non-trivial path is a tree with two end-vertices) and for the complete graph $K_{p}, e d m^{+}\left(K_{p}\right)=p$.

Theorem 2.13. For a connected graph $G, \operatorname{edm}(G)=p$ if and only if $e d m^{+}(G)=p$.

Proof. Let $e d m^{+}(G)=p$. Then $S=V(G)$ is the unique minimal edge detour monophonic set of $G$. Since no proper subset of $S$ is an edge detour monophonic set, it is clear that $S$ is the unique minimum edge detour monophonic set of $G$ and so $\operatorname{edm}(G)=p$. The converse follows from Theorem 2.11 .

Theorem 2.14. If $G$ is a connected graph with $\operatorname{edm}(G)=p-1$, then $e d m^{+}(G)=p-1$. 
Proof. Since $\operatorname{edm}(G)=p-1$, it follows from Theorem 2.11 that $e d m^{+}(G)=p$ or $p-1$. If $e d m^{+}(G)=p$, then by Theorem $2.13, e d m(G)=p$, which is a contradiction. Hence $e d m^{+}(G)=p-1$.

Theorem 2.15. For the complete bipartite graph $G=K_{m, n}$,

(i) $e d m^{+}(G)=2$ if $m=n=1$.

(ii) $e d m^{+}(G)=n$ if $m=1, n \geq 2$.

(iii) $e d m^{+}(G)=\max \{m, n\}$ if $m, n \geq 2$.

Proof. (i) and (ii) follows from Corollary 2.8.

(iii) Let $m, n \geq 2$. Assume without loss of generality that $m \leq n$. Let $X=\left\{x_{1}, x_{2}, \ldots, x_{m}\right\}$ and $Y=\left\{y_{1}, y_{2}, \ldots, y_{n}\right\}$ be the bipartition of $G$. Let $S=Y$. We prove that $S$ is a minimal edge detour monophonic set of $G$. Any edge $x_{i} y_{j}(1 \leq i \leq m, 1 \leq j \leq n)$ lies on the detour monophonic path $y_{j}, x_{i}, y_{k}$ for any $k \neq j$ so that $S$ is an edge detour monophonic set of $G$. Let $S^{\prime} \subset S$. Then there exists a vertex $y_{j} \in S$ such that $y_{j} \notin S^{\prime}$. Clearly, the edge $x_{i} y_{j}$ for every $i$, does not lie on any detour monophonic path joining a pair of vertices in $S^{\prime}$. Thus $S^{\prime}$ is not an edge detour monophonic set of $G$. Hence $S$ is a minimal edge detour monophonic set of $G$ and so $e d m^{+}(G) \geq n$.

Let $S_{1}$ be any minimal edge detour monophonic set of $G$ with $\left|S_{1}\right|>n$. Since any edge $x_{j} y_{i}(1 \leq i \leq n)$ for every $j$, lies on the detour monophonic path $x_{j}, y_{i}, x_{k}$ for $j \neq k$, it follows that $X$ is an edge detour monophonic set of $G$. Hence $S_{1}$ cannot contain $X$. Similarly, since $Y$ is a minimal edge detour monophonic set of $G, S_{1}$ cannot contain $Y$. Hence $S_{1} \subseteq X^{\prime} \cup Y^{\prime}$, where $X^{\prime} \subset X$ and $Y^{\prime} \subset Y$. Hence there exists a vertex $x_{i} \in X(1 \leq i \leq m)$ and a vertex $y_{j} \in Y(1 \leq j \leq n)$ such that $x_{i}, y_{j} \notin S_{1}$. It is easily seen that, the edge $x_{i} y_{j}$ does not lie on any $x-y$ detour monophonic path, for any $x, y \in S_{1}$. Thus $S_{1}$ is not an edge detour monophonic set of $G$, which is a contradiction. Therefore, any minimal edge detour monophonic set of $G$ contains at most $n$ elements so that $e d m^{+}(G) \leq n$. Hence $e d m^{+}(G)=n$.

Theorem 2.16. For any three positive integers $a, b, c$ with $2 \leq a \leq b \leq c$, there is a connected graph $G$ with $m_{1}(G)=a, e d m(G)=b$ and $e d m^{+}(G)=$ $c$.

Proof. Case 1. $2 \leq a=b=c$. Let $G$ be any tree with $a$ end-vertices. Then by Theorem 1.5 and Corollary 2.8, $G$ has the desired properties. 
Case 2. $2 \leq a=b<c$. Let $P_{3}: x, y, z$ be the path of order 3. Let $G$ be the graph obtained by adding $c-1$ new vertices $v_{1}, v_{2}, \ldots, v_{a-1}, w_{1}, w_{2}, \ldots, w_{c-a}$ to $P_{3}$ and joining each $w_{i}(1 \leq i \leq c-a)$ to both $x, z$; and also joining each $v_{i}(1 \leq i \leq a-1)$ to $x$. The graph $G$ is shown in Figure 2.2. Let $S=\left\{v_{1}, v_{2}, \ldots, v_{a-1}\right\}$ be the set of all end-vertices of $G$.

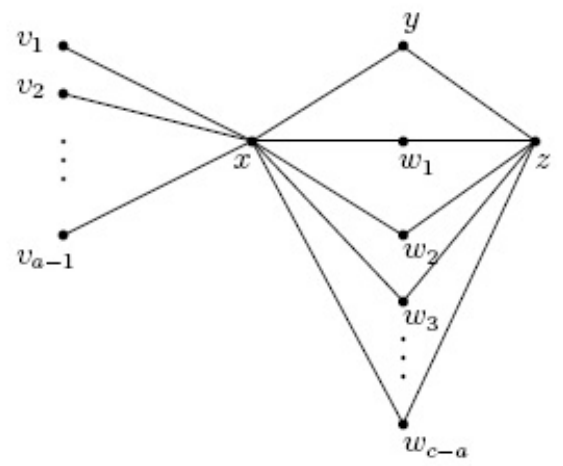

Figure 2.2: $G$

Then by Theorems 1.1, 1.2 and 2.3, $S$ is contained in every edge monophonic set, every edge detour monophonic set and every minimal edge detour monophonic set of $G$. It is clear that $S$ is not an edge monophonic set of $G$. It is easily verified that $S^{\prime}=S \cup\{z\}$ is a minimum edge monophonic set of $G$ and also a minimum edge detour monophonic set of $G$. Thus $m_{1}(G)=e d m(G)=a$.

Next we show that $e d m^{+}(G)=b$. Clearly $T=S \cup\left\{y, w_{1}, w_{2}, \ldots, w_{c-a}\right\}$ is an edge detour monophonic set of $G$. We claim that $T$ is a minimal edge detour monophonic set of $G$. Let $W$ be any proper subset of $T$. Then there exists a vertex, say $v$, such that $v \in T$ and $v \notin W$. By Theorem 2.3, $v \in\left\{y, w_{1}, w_{2}, \ldots, w_{c-a}\right\}$. It is easily verified that the edge $v z$ is not an internal edge of any $x-y$ detour monophonic path for some $x, y \in W$, it follows that $W$ is not an edge detour monophonic set of $G$. Hence $T$ is a minimal edge detour monophonic set of $G$ and so $e d m^{+}(G) \geq c$.

Now, we prove that $e d m^{+}(G)=c$. Suppose that $e d m^{+}(G)>c$. Let $N$ be a minimal edge detour monophonic set of $G$ with $|N|>c$. Then there exists at least one vertex, say $u \in N$ such that $u \notin T$. Then by Theorem 2.7, $u \neq x$ and so $u=z$. Clearly $S \cup\{z\}$ is an edge detour monophonic set of $G$ and it is a proper subset of $N$, which is a contradic- 
tion to $N$ a minimal edge detour monophonic set of $G$. Hence $e d m^{+}(G)=c$.

Case 3. $2 \leq a<b=c$. Let $C_{i}: u_{i}, v_{i}, w_{i}, x_{i}, y_{i}, u_{i}(1 \leq i \leq b-a)$ be $b-a$ copies of a cycle of order 5 . Let $H$ be the graph obtained from $C_{i}(1 \leq i \leq$ $b-a)$ by joining the vertices $w_{i-1}$ of $C_{i-1}$ and $u_{i}$ of $C_{i}(2 \leq i \leq b-a)$. Let $G$ be the graph obtained from $H$ by adding $a$ new vertices $z, z_{1}, z_{2}, \ldots, z_{a-1}$ and (i) joining $z$ to $u_{1}$, (ii) joining each $z_{j}(1 \leq j \leq a-1)$ to $w_{b-a}$. The graph $G$ is shown in Figure 2.3. Let $S=\left\{z, z_{1}, z_{2}, \ldots, z_{a-1}\right\}$ be the set of all extreme vertices of $G$. Then by Theorem 1.1, every edge monophonic set of $G$ contains $S$. Clearly, $S$ is an edge monophonic set of $G$ and so $m_{1}(G)=a$.

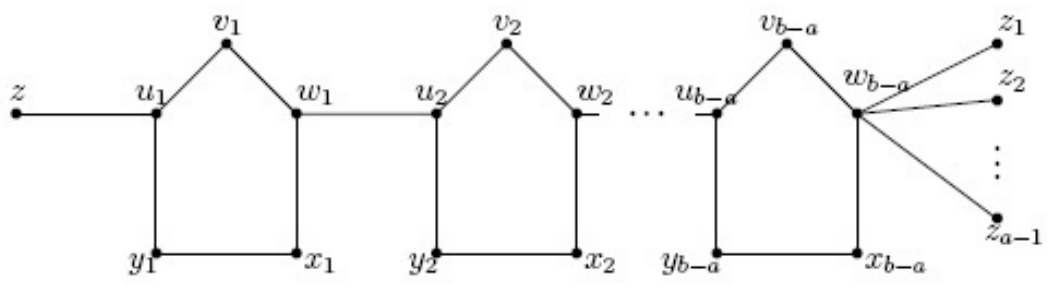

Figure 2.3: $G$

By Theorem 1.2 and Theorem 2.3, every edge detour monophonic set and every minimal edge detour monophonic set of $G$ contains $S$. Clearly, $S$ is not a minimal and minimum edge detour monophonic set of $G$. We observe that every minimal and minimum edge detour monophonic set of $G$ contains exactly one vertex from each set $\left\{v_{i}, x_{i}, y_{i}\right\}$ for every $i(1 \leq$ $i \leq b-a)$. Thus $e d m(G) \geq b$ and $e d m^{+}(G) \geq b$. On the other hand, $S^{\prime}=S \cup\left\{v_{1}, v_{2}, \cdots v_{b-a}\right\}$ is a minimum edge detour monophonic set of $G$, it follows that $\operatorname{edm}(G) \leq b$. Thus $\operatorname{edm}(G)=b$. By Theorem 2.7, no cut-vertex of $G$ belongs to any minimal edge detour monophonic set of $G$. It follows that there does not exist a minimal edge detour monophonic set $N$ of $G$ with $|N|>b$. Hence $e d m^{+}(G)=b$.

Case 4. $2 \leq a<b<c$. Let $V\left(K_{2}\right)=\{x, y\}$ and $V\left(K_{c-b+1}\right)=\left\{l_{1}, l_{2}, \ldots\right.$ $\left.l_{c-b+1}\right\}$. Let $H=\bar{K}_{c-b+1}+\bar{K}_{2}$. Let $C_{i}: u_{i}, v_{i}, w_{i}, x_{i}, y_{i}, u_{i}(1 \leq i \leq b-a)$ be $b-a$ copies of a cycle of order 5 . Let $H^{\prime}$ be the graph obtained from 
$C_{i}(1 \leq i \leq b-a)$ by joining the vertices $w_{i-1}$ of $C_{i-1}$ and $u_{i}$ of $C_{i}(2 \leq i \leq$ $b-a)$. Let $G$ be the graph obtained by joining the vertices $w_{b-a}$ from $H^{\prime}$ and $x$ from $H$, and then adding $a-1$ new vertices $z, z_{1}, z_{2}, \ldots, z_{a-2}$; and (i) joining $z$ to $u_{1}$, (ii) joining $z_{j}(1 \leq j \leq a-2)$ to $x$. The graph $G$ is shown in Figure 2.4. Let $S=\left\{z, z_{1}, z_{2}, \ldots, z_{a-2}\right\}$ be the set of all end-vertices of $G$. Then by Theorem 1.1, every edge monophonic set of $G$ contains $S$. Clearly, $S$ is not an edge monophonic set of $G$. Let $S^{\prime}=S \cup\{y\}$. It is easily verified that $S^{\prime}$ is an edge monophonic set of $G$ and so $m_{1}(G)=a$.

By Theorem 1.2 and Theorem 2.3, every edge detour monophonic set of $G$ and every minimal edge detour monophonic set of $G$ contains $S$. Clearly, $S$ is not an edge detour monophonic set of $G$. We observe that every minimum edge detour monophonic set of $G$ contains $y$ and exactly one vertex from $\left\{v_{i}, x_{i}, y_{i}\right\}$ for every $i(1 \leq i \leq b-a)$. Thus $e d m(G) \geq b$. On the other hand, $S^{\prime}=S \cup\left\{v_{1}, v_{2}, \cdots v_{b-a}, y\right\}$ is a minimum edge detour monophonic set of $G$ and so $\operatorname{edm}(G)=b$.

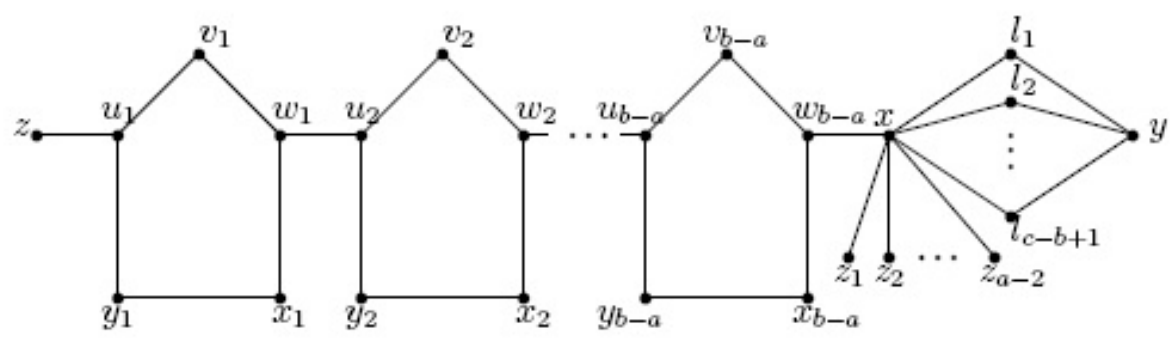

Figure 2.4: $G$

Now, $T=S \cup\left\{v_{1}, v_{2}, \ldots, v_{b-a}, l_{1}, l_{2}, \ldots, l_{c-b+1}\right\}$ is an edge detour monophonic set of $G$. We show that $T$ is a minimal edge detour monophonic set of $G$. Let $W$ be any proper subset of $T$. Then there exists at least one vertex, say $v \in T$, such that $v \notin W$. If $v=v_{i}(1 \leq i \leq b-a)$, the edge $v u_{i}$ is not an internal edge of any $x-y$ detour monophonic path for some $x, y \in W$, it follows that $W$ is not an edge detour monophonic set of $G$. If $v=l_{i}(1 \leq i \leq c-b+1)$, the edge $v y$ is not an internal edge of any $x-y$ detour monophonic path for some $x, y \in W$, it follows that $W$ is not an edge detour monophonic set of $G$. Hence $T$ is a minimal edge detour monophonic set of $G$ and so $e d m^{+}(G) \geq c$. 
Next we show that there is no minimal edge detour monophonic set $X$ of $G$ with $|X| \geq c+1$. Suppose that there exists a minimal edge detour monophonic set $X$ of $G$ such that $|X| \geq c+1$. Then there exists at least one vertex, say, $v \in X$ such that $v \notin T$. We observe that every minimal edge detour monophonic set contains exactly one element from $\left\{v_{i}, x_{i}, y_{i}\right\}$ for every $i(1 \leq i \leq b-a)$. Hence by Theorem 2.7, $v=y$. Clearly, $\left(X-\left\{l_{1}, l_{2}, \ldots, l_{c-b+1}\right\}\right) \cup\{y\}$ is a minimal edge detour monophonic set of $G$, which is a contradiction. Therefore $e d m^{+}(G)=c$.

Theorem 2.17. For any three positive integers $b, c$ and $n$ with $2 \leq b \leq$ $n \leq c$, there is a connected graph $G$ with $\operatorname{edm}(G)=b, e d m^{+}(G)=c$ and a minimal edge detour monophonic set of cardinality $n$.

Proof. We consider four cases.

Case 1. $b=n=c$. Let $G$ be any tree with $b$ end-vertices. Then by Corollary $2.8, G$ has the desired properties.

Case 2. $b=n<c$. For the graph $G$ given in Figure 2.2 of Theorem 2.16, it is proved that $\operatorname{edm}(G)=b, e d m^{+}(G)=c$ and $S=\left\{z, v_{1}, v_{2}, \ldots, v_{b-1}\right\}$ is a minimal edge detour monophonic set of cardinality $n$.

Case 3. $b<n=c$. For the graph $G$ given in Figure 2.2 of Theorem 2.16, it is proved that $e d m(G)=b, e d m^{+}(G)=c$ and $S=\left\{v_{1}, v_{2}, \ldots, v_{b-1}, y\right.$, $\left.w_{1}, w_{2}, \ldots, w_{c-b}\right\}$ is a minimal edge detour monophonic set of cardinality $n$.

Case 4. $b<n<c$. Let $l=n-b+1$ and $m=c-n+1$.

Let $F_{1}=m K_{1}+\overline{K_{2}}$, where $U_{1}=V\left(\overline{K_{2}}\right)=\left\{x, u_{1}\right\}$ and $X=V\left(m K_{1}\right)=$ $\left\{x_{1}, x_{2}, \ldots, x_{m}\right\}$. Similarly, $F_{2}=l K_{1}+\overline{K_{2}}$, where $U_{2}=V\left(\overline{K_{2}}\right)=\left\{u_{2}, y\right\}$ and $Y=V\left(l K_{1}\right)=\left\{y_{1}, y_{2}, \ldots, y_{l}\right\}$. Let $K_{1, b-2}$ be the star at the vertex $u$ and let $S=\left\{w_{1}, w_{2}, \ldots, w_{b-2}\right\}$ be the set of end-vertices of $K_{1, b-2}$. Let $G$ be the graph obtained from $K_{1, b-2}, F_{1}$ and $F_{2}$ by identifying the vertices $u$ from $K_{1, b-2}, u_{1}$ from $F_{1}$ and $u_{2}$ from $F_{2}$. The graph $G$ is shown in Figure 2.5. It follows from Theorem 2.3, every minimal edge detour monophonic set contains $S$. 


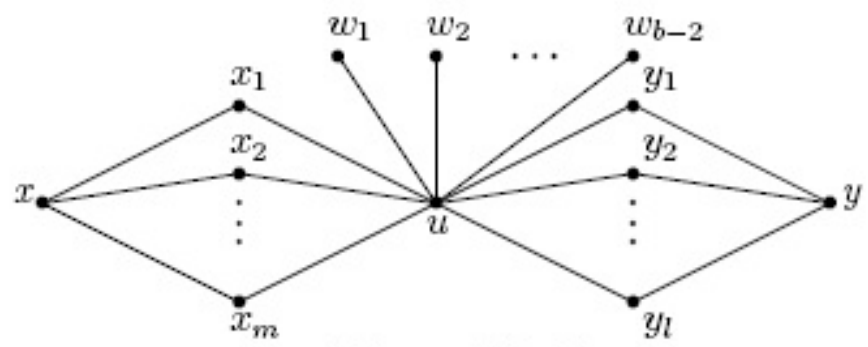

Figure 2.5: $G$

First we show that $e d m(G)=b$. It is clear that $S$ is not an edge detour monophonic set of $G$. Also, for any $v \in V(G)-S, S \cup\{v\}$ is not an edge detour monophonic set of $G$. Let $S^{\prime}=S \cup\{x, y\}$. It is easily verified that $S^{\prime}$ is a minimum edge detour monophonic set of $G$ and so $e d m(G)=b$.

Next, we show that $e d m^{+}(G)=c$. Let $T=S \cup X \cup Y$. It is clear that $T$ is an edge detour monophonic set of $G$. We claim that $T$ is a minimal edge detour monophonic set of $G$. Let $W$ be any proper subset of $T$. Then there exists a vertex, say, $v \in T$ such that $v \notin W$. Assume first that $v=x_{i}$ for some $i(1 \leq i \leq m)$ or $v=y_{j}$ for some $j(1 \leq j \leq l)$. Then the edge $u v$ is not an internal edge of any detour monophonic path joining a pair of vertices in $W$. If $v=w_{i}$ for some $i(1 \leq i \leq b-2)$, then the edge $u w_{i}$ is not an internal edge of any $x-y$ detour monophonic path for some $x, y \in W$. Hence $T$ is a minimal edge detour monophonic set of $G$ and so $e d m^{+}(G) \geq|T|=b-2+l+m=c$.

Now, we prove that $e d m^{+}(G)=c$. Suppose that $e d m^{+}(G)>c$. Let $T^{\prime}$ be a minimal edge detour monophonic set of $G$ with $\left|T^{\prime}\right|>c$. Then there exists at least one vertex, say $v \in T^{\prime}$ such that $v \notin T$. Also, by Theorem 2.7, $v \in\{x, y\}$. If $v=x$, then $T^{\prime}-X$ is an edge detour monophonic set of $G$ and it is a proper subset of $T^{\prime}$, which is a contradiction to $T^{\prime}$ a minimal edge detour monophonic set of $G$. Similarly, if $v=y$, then $T^{\prime}-Y$ is an edge detour monophonic set of $G$ and it is a proper subset $T^{\prime}$, which is a contradiction. Hence $e d m^{+}(G)=c$.

Next we show that there is a minimal edge detour monophonic set of cardinality $n$. Let $P=\left\{w_{1}, w_{2}, \ldots, w_{b-2}, x, y_{1}, y_{2}, \ldots, y_{l}\right\}$. It is clear that $P$ is an edge detour monophonic set of $G$. We claim that $P$ is a minimal 
edge detour monophonic set of $G$. Assume, to the contrary, that $P$ is not a minimal edge detour monophonic set of $G$. Then there is a proper subset $P^{\prime}$ of $P$ such that $P^{\prime}$ is an edge detour monophonic set of $G$. Let $v \in P$ and $v \notin P^{\prime}$. By Theorem 1.2, clearly $v=x$ or $v=y_{i}$ for some $i=1,2, \ldots, l$. If $v=x$, then the edges $v x_{j}$ and $x_{j} u(1 \leq j \leq m)$ are not internal edges of any $s-t$ detour monophonic path for some $s, t \in P^{\prime}$. If $v=y_{i}$ for some $i=1,2, \ldots l$, then the edge $v u$ is not an internal edge of any $s-t$ detour monophonic path for some $s, t \in P^{\prime}$. Hence $P$ is a minimal edge detour monophonic set of $G$ with cardinality $|P|=n$.

\section{References}

[1] F. Harary, Graph Theory, Addison-Wesley, (1969).

[2] A. P. Santhakumaran, P. Titus and P. Balakrishnan, Some Realisation Results on Edge Monophonic Number of a Graph, communicated.

[3] A. P. Santhakumaran, P. Titus, K. Ganesamoorthy and P. Balakrishnan, Edge Detour Monophonic Number of a Graph, Proyecciones Journal of Mathematics, Vol. 32, No. 2, pp. 183-198, (2013).

[4] P. Titus, K. Ganesamoorthy and P. Balakrishnan, The Detour Monophonic Number of a Graph, J. Combin. Math. Combin. Comput. 83, pp. 179-188, (2013).

[5] P. Titus and K. Ganesamoorthy, On the Detour Monophonic Number of a Graph, Ars Combinatoria, to appear.

\section{P. Titus}

Department of Mathematics

University College of Engineering Nagercoil

Anna University, Tirunelveli Region

Nagercoil - 629 004,

India

e-mail: titusvino@yahoo.com

and 
K. Ganesamoorthy

Department of Mathematics

University V.O.C. College of Engineering

Anna University, Tirunelveli Region

Tuticorin - 628 008,

India

e-mail : kvgm_2005@yahoo.co.in 